\title{
Effervescence in a glass of champagne:
}

\section{A bubble story}

Gérard Liger-Belair* and Philippe Jeandet

Laboratoire d'oenologie, faculté des sciences de Reims, France

*: Corresponding author, e-mail: gerard.liger-belair@univ-reims.fr

$D^{\text {eople have long been fascinated by bubbles and foams }}$ $P$ dynamics, and since the pioneer work of Leonardo da Vinci in the early $16^{\text {th }}$ century, this subject has generated a huge bibliography. However, only very recently, much interest was devoted to bubbles in champagne wines [1]. Small bubbles rising through the liquid, as well as a bubble ring (the so-called collar) at the periphery of a flute poured with champagne, are the hallmark of this traditionally festive wine, and even if there is no scientific evidence yet to connect the quality of a champagne with the fineness of its bubbles, people nevertheless often make a connection between them. Therefore, since the last few years, a better understanding of the role played by each of the numerous parameters involved in the bubbling process has become an important stake in the champagne research area. Furthermore, in addition to these strictly enological reasons, we also feel that the area of bubble dynamics and especially the area of collapsing bubble dynamics could benefit from the simple but close observation of a glass poured with champagne.

In this paper, our first results concerning the close observation of the three main steps of a champagne bubble's life are summarised, i.e. the bubble nucleation, the bubble ascent and the collapse of a bubble bursting at the free surface of the liquid. Our results were obtained in real consuming conditions, in a classical crystal flute poured with a standard commercial Champagne wine.

\section{Bubble nucleation}

In the case of Champagne wines, the main gas responsible for bubble production is carbon dioxide, which is produced by yeasts during the second fermentation in the closed bottle. According to Henry's law, equilibrium progressively establishes between the gas dissolved into the wine and the gas into the vapour phase in the headspace under the cork. At the end of fermentation, the $\mathrm{CO}_{2}$ pressure under the cork is around $6 \mathrm{~atm}$., and the wine may contain up to $12 \mathrm{~g} / \mathrm{L}$ of dissolved $\mathrm{CO}_{2}$. When the bottle is opened, the $\mathrm{CO}_{2}$ pressure in the vapour phase suddenly falls. The thermodynamic equilibrium of the closed bottle is broken, and the wine becomes supersaturated with $\mathrm{CO}_{2}$ molecules. To recover a new stable thermodynamic state corresponding to the atmospheric pressure, champagne must degas. When champagne is poured into a glass, two mechanisms enable dissolved $\mathrm{CO}_{2}$ molecules to escape from the supersaturated liquid medium: diffusion through the flat free surface of the liquid, and bubble formation. As soon as a liquid medium is supersaturated, the bulk free energy per unit of volume, $\Delta g v$, associated with the transfer of dissolved gas molecules into the vapour is negative, and therefore thermodynamically favourable. But the bubble production process also results into the production of interfacial free energy. Below a critical radius $r_{c}$ depending on some physicochemical parameters of the solution, the bubble embryo formation results in a net increase of the total free energy of the system. Henceforth, classical nucleation is characterized by an energy barrier to

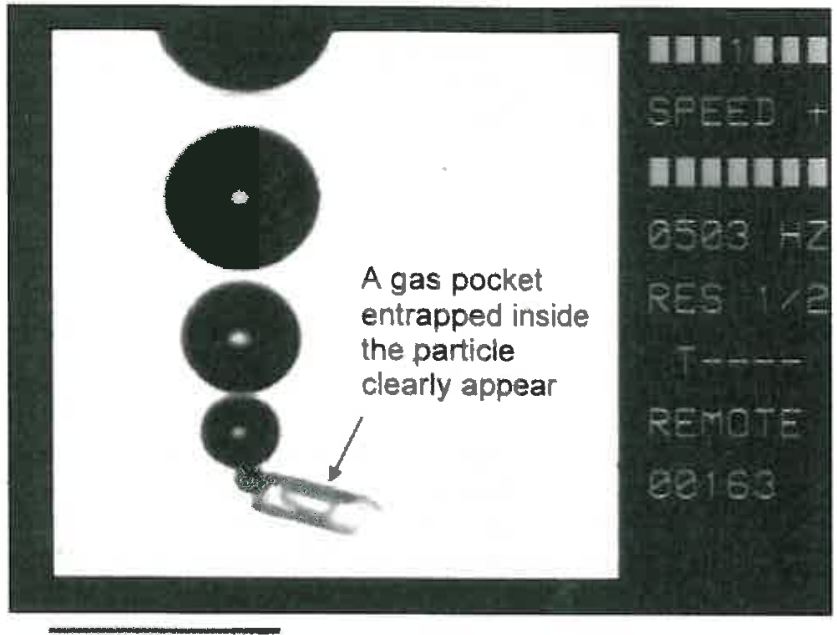

$100 \mu \mathrm{m}$

Fig. 1: Close-up of a particle acting as a nucleation site on the wall of a glass poured with champagne. Most of these particles are hollow elongated and roughly cylindrical fibres.

overcome [2]. Therefore, homogeneous bubble nucleation within the liquid bulk or heterogeneous nucleation on smooth surfaces requires very high supersaturating ratios, which are totally unrealistic in the case of Champagne wines [3,4]. In weakly supersaturated liquids, such as champagne, sparkling wines and carbonated beverages in general, bubbles need pre-existing gas cavities with radii of curvature greater than the critical radius in order to overcome the nucleation energy barrier and grow freely. In this type of non-classical heterogeneous nucleation, dissolved gas molecules spontaneously diffuse through the meniscus of pre-existing gas cavities.

Contrary to a generally accepted idea, nucleation sites are not located on irregularities of the glass itself. The length-scale of glass and crystal irregularities is far below the critical radius of curvature required for the non-classical heterogeneous nucleation. Most of nucleation sites are located on hollow and roughly cylindrical exogenous cellulose fibres coming from the surrounding air or remaining from the wiping process. Because of geometrical and hydrophobic properties, such particles are able to entrap gas pockets during the filling of a flute and thus to start up the bubble production process. A typical nucleation site is displayed in figure 1. The gas pocket entrapped into the particle and which starts-up the bubble production process clearly appears. Such particles are responsible for the clockwork and repetitive production of bubbles that rise in-line into the form of elegant bubble trains [5-7]. This cycle of bubble production at a given nucleation site is characterised by its "bubbling" frequency. The 
time needed to reach the moment of bubble detachment depends on the kinetics of the $\mathrm{CO}_{2}$ molecules transfer from the champagne to the gas pocket, but also on the geometrical properties of the given nucleation site. Now, since a collection of particle shapes and sizes exists on the glass wall, the bubbling frequency may also vary from one site to another. Three minutes after pouring, we measured frequencies ranging from less than $1 \mathrm{~Hz}$ up to almost $30 \mathrm{~Hz}$, which means that the most active nucleation sites emit up to 30 bubbles per second.

\section{Bubble rise}

A short examination of the typical regular bubble train presented in figure 2 is already very instructive. It appears clearly that bubbles grow during ascent. Moreover, as the distance between two successive bubbles increases, and since bubbles are released from the nucleation site with clockwork regularity, it can be guessed that a bubble accelerates when rising through the liquid. In order to better analyse hydrodynamic and physicochemical parameters that control bubble ascent, let us write the equation of motion of an expanding spherical gas bubble, rising in a low viscous fluid, as in the case of champagne bubbles. After release from its nucleation site, a bubble experiences, in addition to the buoyancy

$$
F_{B}=\rho g \frac{4}{3} \pi R^{3}
$$

a viscous drag force exerted by the surrounding fluid. This drag force is classically expressed by

$$
F_{D}=C_{D} \frac{\rho U^{2}}{2} \pi R^{2}
$$

where $C_{D}$ is a dimensionless drag coefficient ${ }^{\dagger}$. Inertia of bubbles can obviously be neglected, but during the rise, bubbles induce a displacement of the surrounding fluid in their vicinity, which leads to an added-mass force. The added-mass of a bubble is

$$
M_{0}=C_{A M} \rho \frac{4}{3} \pi R^{3}
$$

where the added-mass coefficient $C_{A M}$ is the ratio of the surrounding volume of liquid displaced during ascent to the bubble volume. The equation of motion of champagne rising and expanding gas bubbles can therefore be written under the form,

$$
\frac{d}{d t}\left(M_{0} U\right)=\frac{4}{3} \rho g \pi R^{3}-C_{D} \frac{1}{2} \rho U^{2} \pi R^{2}
$$

Since the bubble radius expands during the rise, this equation reduces to,

$$
C_{A M} \rho \frac{4}{3} \pi R^{3}\left(\frac{d U}{d t}+\frac{3 U}{R} \frac{d R}{d t}\right)=\frac{4}{3} \rho g \pi R^{3}-C_{D} \frac{1}{2} \rho U^{2} \pi R^{2}
$$

It was nevertheless found that the added-mass effect of a bubble approaching the free surface never exceeds $2-3 \%$ of its buoyancy [5-7]. As a result, it will be neglected in the following. Thus, the

\footnotetext{
† $R$ is the bubble radius, $U$ is the rising velocity, $\rho$ is the liquid density,
} and $g$ is the acceleration due to gravity.
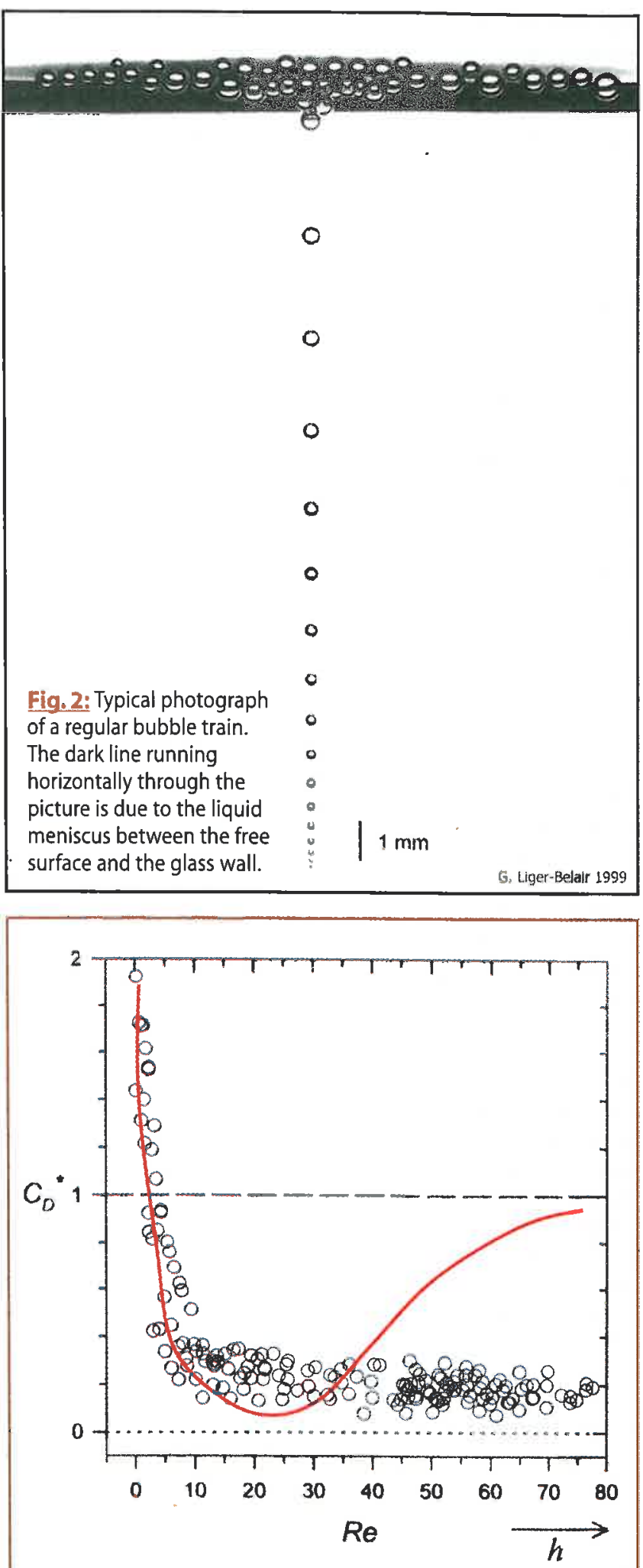

Fig.3: Normalised drag coefficient experienced by champagne bubbles during ascent as a function of the Reynolds number $R e$ (and indirectly as a function of the travelled distance $h$ from the nucleation site); fluid sphere limit (--- -); rigid sphere limit (- -); normalised drag coefficient that a bubble of fixed radius would experience $(-)$. 
equation of motion (2) reduces itself to the classical balance between drag force and buoyancy. Now, for each bubble of a given bubble train, the experimental determination of $R$ and $U$ leads to the experimental determination of the drag coefficient $C_{D}$ along the rise, through the expression,

$$
C_{D}=\frac{8 g R}{3 U^{2}}
$$

During the last decades, many empirical or semi-empirical equations have been proposed to approximate $C_{D}$ for bubbles in free rise. Some of the most popular are listed in the book of Clift et al. [8]. Our measurements were compared with two of them, respectively $C_{R S}$ and $C_{F S}$, available in the whole range of Reynolds numbers $R e$ covered by champagne bubbles. $C_{R S}$ concerns rigid spheres, and is applicable for rising bubbles completely covered with surfactants, whereas $C_{F S}$ was obtained for fluid spheres, i.e. bubbles with a fully mobile interface free from surface-active materials.

In order to indirectly access the bubble surface state during the rise, the normalised drag coefficient $C_{D}^{*}$ defined as follows was used,

$$
C_{D}^{*}=\frac{\left(C_{D}-C_{F S}\right)}{\left(C_{R S}-C_{F S}\right)}
$$

Bubbles with a fully mobile interface behaving hydrodynamically as fluid spheres will exhibit values of $C_{D}^{*}$ close to zero, whereas polluted bubbles behaving hydrodynamically as rigid spheres will have values of $C_{D}^{*}$ close to one. In figure 3 , the normalised drag coefficient of champagne bubbles was plotted as a function of $\mathrm{Re}$, for bubbles of various bubble trains. After a first regime attributed to both inertia and wall effects where $C_{D}^{*}>1$, it appears clearly from figure 3 that bubbles reach a quasi-stationary stage intermediate between that of a rigid and that a fluid sphere (but nevertheless closer to that of a fluid sphere). This result drastically differs from the result classically observed with bubbles of fixed radii rising in surfactant solutions. Actually, surfactants progressively adsorb at the surface of a rising bubble thus increasing the immobile area of the bubble surface. Therefore, the drag coefficient experienced by a rising bubble of fixed radius progressively increases, and inexorably reaches the rigid sphere limit when the bubble interface gets completely contaminated. Strictly speaking, the complete immobilisation of the interface of a bubble rising in a surfactant solution is reached before its complete coverage, as demonstrated by Ybert and di Meglio [9]. In the case of a champagne bubble, since the bubble expands during its rise through the supersaturated liquid, the bubble interface continuously increases, and therefore continuously offers newly created surface to the adsorbed surface-active materials (around $5 \mathrm{mg} / \mathrm{L}$, mostly composed of proteins and glycoproteins). Expanding bubbles experience two opposing effects. Figure 3 suggests that the bubble growth during ascent approximately balances the adsorption rate of surface-active compounds on the rising bubble.

We also compared the behaviour of champagne bubbles with that of beer bubbles. It was found that beer bubbles showed a behaviour, very close to that of rigid spheres [5-7], thus confirming a previous study [10]. This is not a surprising result, since beer contains much higher amounts of surface-active macromolecules (of order of several hundreds $\mathrm{mg} / \mathrm{L}$ ) likely to be adsorbed at a bubble interface than champagne. Furthermore, since the gas content is lower in beer, growth rates of beer bubbles are lower than those of champagne. As a result, the dilution effect due to the rate of dilatation of the bubble area may be too weak to avoid the rigidification of the beer bubble interface.

\section{Bubble collapse at the free surface}

We report now results concerning bubbles collapsing at the free surface of champagne. A reconstructed time sequence illustrating four stages of the collapse of a single bubble is presented in figure 4. Photographs reproduced here were taken immediately after the rupture of a bubble cap [11]. A brief description of each frame follows.
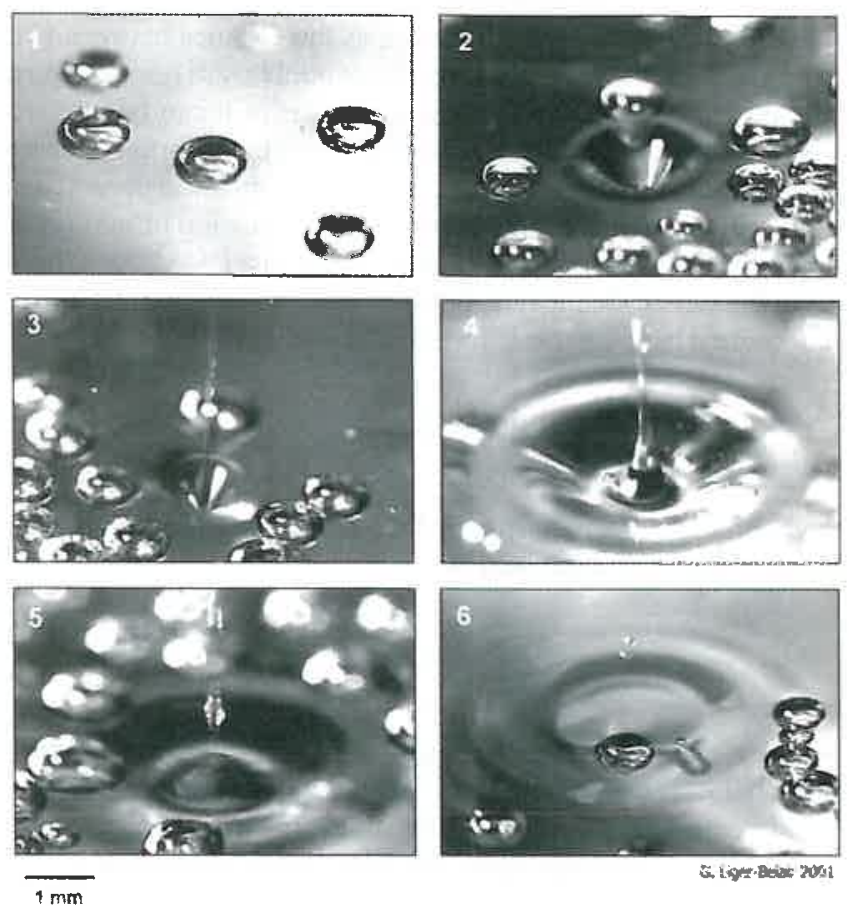

Fig. 4: Reconstructed time sequence illustrating six stages of the collapse of a single bubble at the free surface. The time interval between each frame is around $1 \mathrm{~ms}$.

Between the frame 1 and 2, the thin liquid film, which constitutes the emerged part of the bubble, has just ruptured (on a time-scale of 10 to $100 \mu \mathrm{s}$ ). During this extremely brief initial phase, the bulk shape of the bubble has been frozen. A nearly millimetric open cavity remains at the free surface. While collapsing, the bubble cavity gives rise to a high-speed liquid jet above the free surface (frames 3 and 4). Near the base of the liquid jet, one can distinguish an extremely small bubble (around $100 \mu \mathrm{m}$ ), probably entrapped during the collapsing process. Due to its own velocity, this upward liquid jet becomes unstable and breaks up into droplets called jet drops (frame 5). The combined effects of inertia and surface tension give droplets various and often amazing shapes. Finally, droplets ejected by the parent bubble recover a quasi spherical shape (frame 6). Due to surface excitations following bubble collapse, capillary wave trains centred on the bursting bubble are propagating at the free surface. On the right side of the central bubble, the tiny bubble entrapped during collapse can be observed.

The liquid jet that follows a bubble collapse strikingly resemble, in miniature, that one can observe as a drop impacts the 
surface of a pool of liquid. In his book "A Study of Splashes", Worthington, presented remarkably sharp photographs of drops impacts [12]. Shape details of two various liquid jets, produced during a bubble collapse and during a drop impact are displayed in figure 5. Hydrodynamic structures arising after a drop impact are clearly very close to those which follow a bubble collapse. Since hundreds of bubbles are bursting every second during the first minutes of champagne-tasting, one can conclude that the free surface of a glass of champagne is literally spiked with such cone-shaped liquid structures, unfortunately too short-lived to be observed to the naked eye.

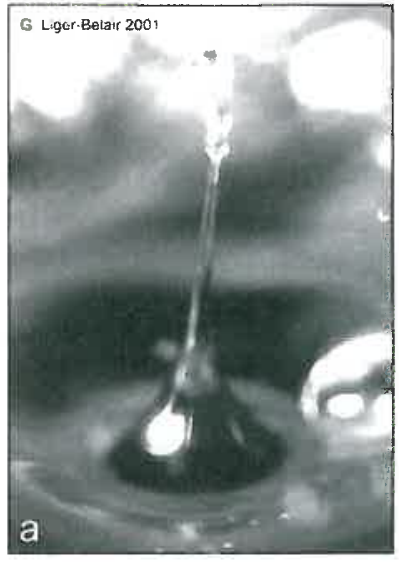

$1 \mathrm{~mm}$

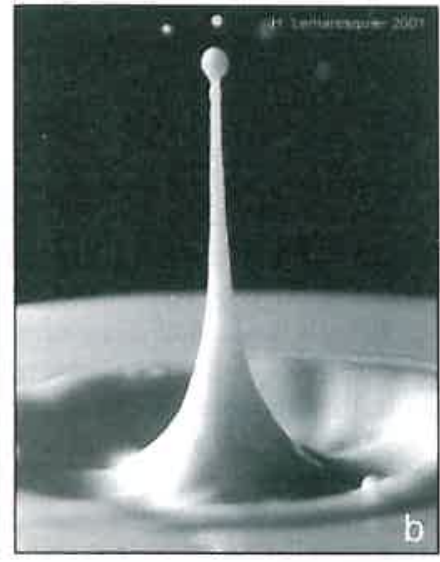

$1 \mathrm{~cm}$
Fig. 5: Close-ups of two liquid jets that follow respectively, a bubble collapse (a), and a drop impact (b).

At a millimetric scale, such a violent hydrodynamic phenomenon which leads to the projection of a high-speed liquid jet is driven by the capillary pressure gradients arising in the layer around the open cavity left by a bursting bubble. Immediately after the rupture of the bubble cap, the sides of the open cavity becomes a region of positive curvature. It ensues a ring of high pressure on the sides of the open cavity. At the same time, due to a negative curvature, a low pressure zone exists around the underside of the cavity. As a result, fluid is rapidly drawn from the sides to the axis of symmetry. The underside of the cavity becomes a region of high pressure. This pushes fluid upward and downward to produce two liquid jets.

Since the first photographic investigation published about fifty years ago [13], numerous experiments have been conducted with single bubbles collapsing at a free surface. But, to the best of our knowledge, and surprising as it may seem, no results concerning the collateral effects on adjoining bubbles of bubbles collapsing in a bubble monolayer have been reported up to now. Actually, effervescence in a glass of champagne ideally lends to a preliminary work with bubbles collapsing in a bubble monolayer. For a few seconds after pouring, the free surface is completely covered with a monolayer composed of quite monodisperse millimetric bubbles collapsing close to each others. Photographs displayed in figure 6 were taken immediately after the rupture of a bubble cap. Adjoining bubble-caps are literally sucked toward the lowest part of the cavity left by the bursting bubble, leading to unexpected and short-lived
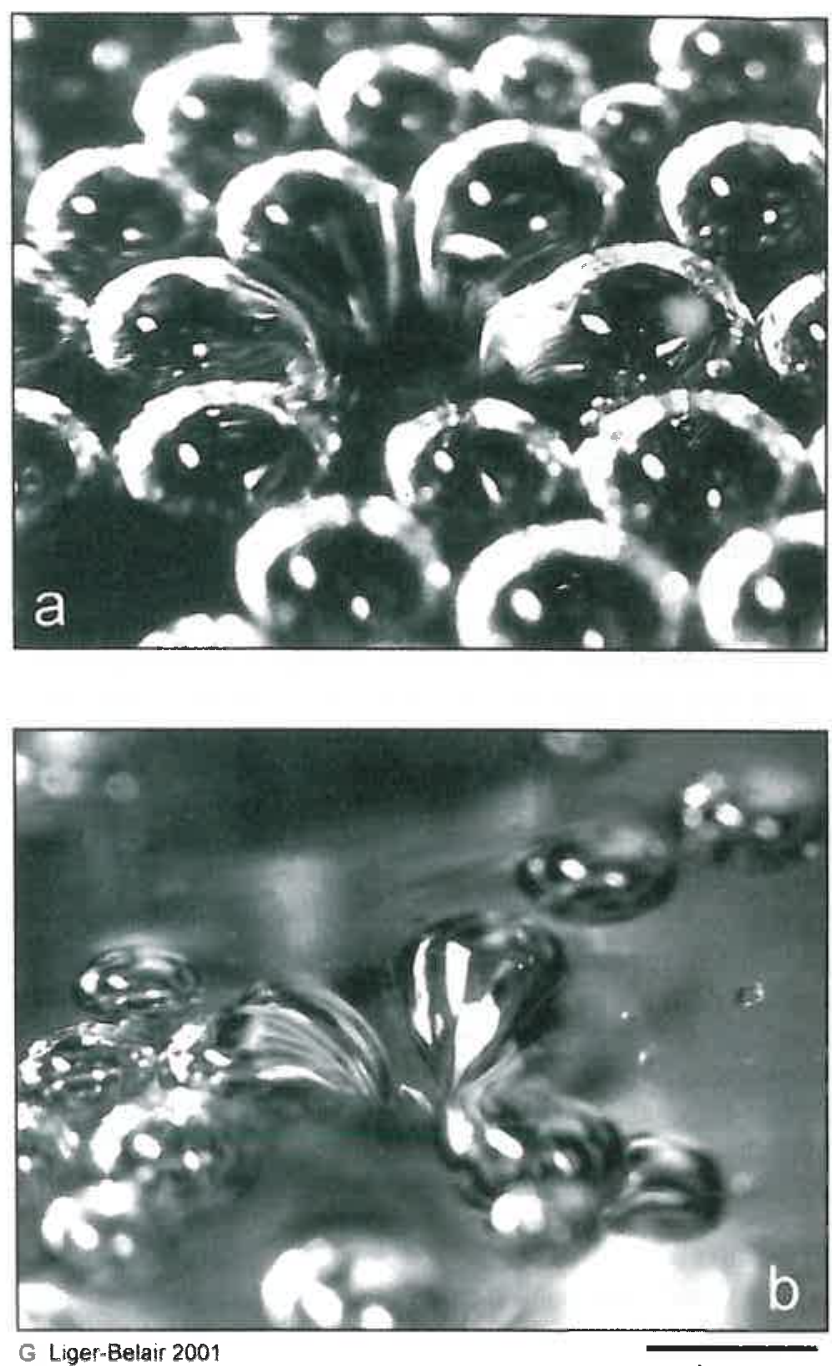

$1 \mathrm{~mm}$

Fig. 6: Oblique view of the collateral effects on adjoining bubbles of a bubble collapsing at the free surface.

flower-shaped structures [14]. During the stretching process, energy is supposed to be mainly stored as surface free energy. A systematic image analysis of numerous time sequences conducted with an high-speed video camera demonstrated an average increase $\triangle A$ of $15 \%$ within approximately $300 \mu$ s of bubble areas adjacent to collapsing bubbles. During the stretching process, stresses in a distorted bubble-cap (one petal of the flower-shaped structure) can be evaluated as shown below in equation 5 .

By comparison, in a previous study, a numerical model conducted to stresses of the order of $10^{4} \mathrm{dyn} \mathrm{cm}^{-2}$ in the boundary
Equation $5^{+t}$

$\left[\frac{\Delta E}{V}\right]_{\text {bubble-cap }} \approx \frac{2 \gamma \Delta A}{A e} \approx \frac{0.15 \times 2 \gamma}{e} \approx 10^{4}-10^{5} \mathrm{~J} / \mathrm{m}^{3}=10^{5}-10^{6} \mathrm{dyn} / \mathrm{cm}^{2}$

${ }^{\text {t+ }} \Delta E$ is the corresponding surface energy in excess during the stretching process, $V$ is the volume of liquid in the emerging bubble-cap, $\gamma$ is the champagne surface tension ( $\approx 50 \mathrm{mN} / \mathrm{m}$ ), $A$ is the emerging bubble area, and $e$ is the thickness of the thin liquid film of the bubble-cap (of order of $10^{-6}-10^{-7} \mathrm{~m}$ ) [15]. 
layer around an isolated millimetric collapsing bubble [16]. This is a brand-new and slightly counter-intuitive result. While absorbing the energy released during collapse, as an air-bag would do, adjoining bubble store this energy into the thin liquid film of emerging bubble-caps, leading finally to stresses higher than those observed in the boundary layer around single millimetric collapsing bubbles. Further investigation should be conducted now, and especially numerically, in order to better understand the relative influence of each pertinent parameters (bubble size, liquid density and viscosity, effect of surfactant...) on bubble deformation.

Contrary to what could have been thought at first glance, effervescence of champagne turned out to be a fantastic tool to investigate a first approach of the physical chemistry of rising and collapsing bubble dynamics. As we do, we hope that the reader will now look at the effervescence in this traditionally festive wine, not only as a nice visually appealing phenomenon, but also as a very instructive one in terms of bubble dynamics study. It would have been regrettable not to have a closer look at such a daily phenomenon, before considering a more academic approach under certainly better controlled, but also less seducing conditions.

\section{Acknowledgments}

Thanks are due to the Europôl'Agro institute and to Jean-Claude Colson and his association "Recherche Oenologie Champagne Universite" for financial support, to Champagne Moët \& Chandon and Pommery for supplying wines, and to Verrerie Cristallerie d'Arques.

The above article is an updated version of a previous article published in the "Bulletin de la Société Française de Physique" vol. 127,pp. 9-11, (2000/2001).

\section{References}

[1] M.Vignes-Adler, Bulletin de la S.F.P. (Société Française de Physique) 106,27 (1996)

[2] M. Blander, Adv. Colloid Interface Sci. 10, 1 (1979)

[3] P. M. Wilt, J. Colloid and Interface Sci. 112, 530 (1986)

[4] S. E. Jones, G. M. Evans, K. P. Galvin, Adv. Colloid Interface Sci. 80, 27 (1999)

[5] G. Liger-Belair et al., Langmuir 16, 1889 (2000)

[6] G. Liger-Belair, Bulletin de la S.F.P. 127, 9 (2000/2001)

[7] G. Liger-Belair, Une première approche des processus physicochimiques liés à l'effervescence des vins de Champagne, $\mathrm{PhD}$ Thesis, University of Reims, France (2001)

[8] R. Clift, J. R. Grace, M. E. Weber, Bubbles, Drops and Particles, Academic Press, New York, (1978)

[9] C. Ybert and J.-M. di Meglio, Eur. Phys. J. B. 4, 313 (1998)

[10] N. Shafer and R. Zare, Physics Today 44, 48 (1991)

[11] G. Liger-Belair et al., Am. J. Enol. Vitic. 52, 88 (2001)

[12] A. M. Worthington, A study of splashes, Longman \& Green ed., London, (1908)

[13] A. H. Woodcock, C. F. Kientzler, A. B. Arons, D. C. Blanchard, Nature 172,1144 (1953)

[14] G. Liger-Belair, M. Vignes-Adler, B. Robillard, P. Jeandet, C. R. Acad. Sci. Paris série IV (Physique-Astrophysique) 2, 775 (2001)

[15] J.Senée, B. Robillard, M.Vignes-Adler, Food Hydrocolloids 13, 15 (1999)

[16] J. M. Boulton-Stone and J. R. Blake, J. Fluid Mech. 254, 437 (1993). 